\title{
Finite element analysis of the fan blade in the Creo Simulation Environment
}

\author{
Fujian Yang ${ }^{1, \text { a }}$, Zhe Chen ${ }^{2}$ \\ ${ }^{1}$ Guilin University of Aerospace Technology, Guilin 541004, China \\ ${ }^{2}$ Sichuan Engineering Technical College, Deyang 618099, China \\ askeen2007@126.com
}

Keywords: fan blade, Creo Simulate, Finite element analysis

\begin{abstract}
The fan blade is the key component of the whole engine, using digital modeling software to reverse the structure model of fan blade, and the Finite element analysis, parameter sensitivity research and optimization design of fan blades are carried out by Creo Simulate. The load of the blade under the corresponding working condition is obtained, the stress distribution of the blade is calculated, and the influence of load on the stress and strength of the blade is analyzed. The results show that the stress concentration position of the blade mainly appears in the middle of the blade, but they all meet the requirements of the structural strength design standard.
\end{abstract}

\section{Introduction}

The fan blade is located at the front of the engine, one of the main working parts of the engine, which provides about $80 \%$ of the thrust for the engine ${ }^{[1]}$. Under the environment of high speed, pneumatic, vibration and other complex hybrid loads for a long time, its strength and reliability have a direct impact on the safety of the engine. In the process of working, the fan blades are subjected to centrifugal force caused by high speed rotation, and are also subjected to complex aerodynamic forces. Therefore, considering the two loads of centrifugal and aerodynamic forces, the stress distribution and strength analysis of fan blades under critical conditions are studied ${ }^{[2]}$.

This paper based on the Creo software and established a three-dimensional model of a certain type of engine fan blade by the software. Considering the stress of fan blades under centrifugal load, the finite element analysis of fan blades is carried out ${ }^{[3]}$. The stress analysis of blades under critical conditions is carried out through Creo Slmulate. The influence of load on the stress distribution of the blade is studied, and the strength of the blade is analyzed according to the strength theory.

\section{Finite element stress analysis and calculation of fan blades}

\subsection{The theory of fan blade strength analysis}

The deformation of the blade at work is small,In general, the size of the deformation is not predetermined in advance and can only be estimated by experience. From the existing engine, the fan blade has smaller deformation, which can be considered to meet the requirements of small deformation; When working, the blade material has not yielded, that is, within the elastic range. At present, most of the engine blades are designed with elastic design, which can meet this requirement; The blades are not in contact with or in contact with other parts. When the blade is discretized, in areas with large stress changes, such as the leaf root, the grid should be classified more closely, and in the area where the stress changes are small, such as the tip of the leaf, the grid can be divided. It should also be noted that when the unit is partitioned, the size of each direction should be approximately the same, and the angle of each element should not be different as far as possible, so as to ensure that the units divided have good characteristics.

The statics calculation equation of structural strength finite element is as follows ${ }^{[4]}$ :

$$
[K]\{u\}=\{F\}
$$




$$
\{\sigma\}=[B][D]\{\mathbf{u}\}
$$

In the formula: $\{u\},\{\sigma\},\{F\}$ They are node displacement, stress and external force, $[K],[B]$, $[D]$ They are stiffness matrix, elastic matrix and strain matrix,

According to the fourth strength theory, the equivalent stress is calculated:

$$
\sigma_{\mathrm{e}}=\sqrt{\frac{1}{2}\left[\left(\sigma_{1}-\sigma_{2}\right)^{2}+\left(\sigma_{2}-\sigma_{3}\right)^{2}+\left(\sigma_{3}-\sigma_{1}\right)^{2}\right]}
$$

In the formula: $\sigma_{\mathrm{e}}$ is Equivalent stress, $\sigma_{1}, \sigma_{2}, \sigma_{3}$ are the first principal stress, the second principal stress and the third main stress.

\subsection{Three dimensional model of fan blade and application of material}

Creo software can achieve seamless integration of geometric 3D modeling and finite element analysis. Because Croe simulate can finish the finite element analysis and modify the 3D model directly in this software, It makes the designer understand the performance of the product earlier in the development cycle, and realize the optimization function at the side of the design. The model set up by the fan blade in the 3D design software Creo is shown in Figure 1.

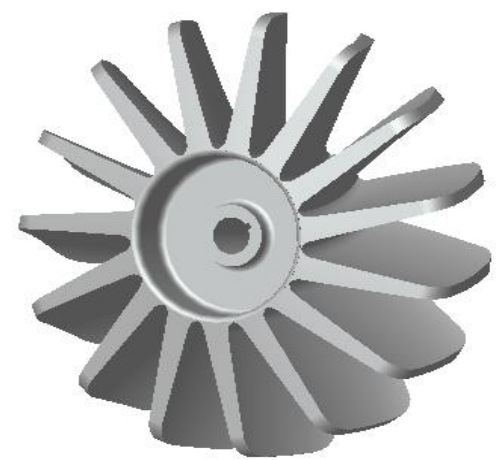

Fig.1 Three dimensional model of fan blade

The material used in the calculation is steel, and the material parameters are shown in Table 1.

Table 1. Physical parameters of titanium alloy(Ti-6Al-4V)

\begin{tabular}{|cc|}
\hline Material & titanium alloy(Ti-6Al-4V) \\
\hline Young's modulus & $1.07 \mathrm{E}+05 \mathrm{MPa}$ \\
Poisson's ratio & 0.29 \\
Density & $4.25 \mathrm{E}+03 \mathrm{~kg} / \mathrm{m}^{3}$ \\
Yield strength & $7.25 \mathrm{E}+02 \mathrm{MPa}$ \\
Tensile strength & $9.75 \mathrm{E}+02 \mathrm{MPa}$ \\
\hline
\end{tabular}

\subsection{Finite element mesh of fan}

In order to better reflect the thin-walled characteristics of the blade, The 20 node hexahedral element is used to mesh the blade and its tenon section ${ }^{[5]}$. The unit size of the fan is $50 \mathrm{~mm}$, due to the need to calculate the strength of the fan blade, so the unit size of the blade's grid fan blade is $20 \mathrm{~mm}$. The grid division uses the self - partition tool in Creo software to generate the finite element mesh for the three-dimensional entity, and obtains 46403 units and 15481 nodes.

\section{The analysis and calculation of the stress and strength of the fan blade.}

\subsection{The stress analysis of the fan blade.}

The blade is the rotating part of the fan and the only element that the fan sends to the energy of the gas. The strength of the fan blade can be judged by the finite element analysis. The fan blade revolves around the axis of the engine at work, and the load is included in the centrifugal force, aerodynamic force and thermal load. Aerodynamic force is a kind of surface distribution pressure, which acts on the 
surface of the blade, but it is not uniform distribution force. In general, aerodynamic force should be taken into consideration when analyzing the stress of a blade, but sometimes it may not be considered because of the lack of data. The centrifugal stress on the fan blade is much larger than the aerodynamic stress, so neglecting the aerodynamic force has little effect on the analysis results.

Centrifugal force is a kind of volume force, which is proportional to the mass and its radius, and it is proportional to the square of the speed. The centrifugal force mainly causes the blade to produce the radial tensile stress, and the torsional stress will be generated for the torsional blade; All blades have thermal loads, but for fan blades, Because of low temperature and uniform distribution of temperature, the thermal stress is small, so it is often ignored in stress analysis. In this paper, the strength of the fan blade is calculated and analyzed under the centrifugal load, and the deformation and stress of the fan blade are calculated by Creo Simulate. The rated speed of the fan is $14400 \mathrm{r} / \mathrm{min}$, in order to analyze the stress distribution of the fan at different speeds. Here are three different speeds of $8000 \mathrm{r} / \mathrm{min}$, $14400 \mathrm{r} / \mathrm{min}$, and $18000 \mathrm{r} / \mathrm{min}$ for analysis.

\subsection{The stress analysis results of fan blades}

As can be seen from fig. 2 and 3, the stress of the fan is concentrated at the root of the fan blade at $14400 \mathrm{r} / \mathrm{min}$.

The maximum stress of the blade is $375.4 \mathrm{MPa}$, the deformation displacement occurs at the tip of the blade of the fan, and the maximum radial displacement of the blade tip is about $2.55 \mathrm{~mm}$. In the same way, the stress of the fan is concentrated at the root of the fan blade at $18000 \mathrm{r} / \mathrm{min}$, and the maximum stress is $525.2 \mathrm{MPa}$. The deformation displacement appears at the tip of the blade of the fan, and the maximum radial displacement of the tip of the leaf is about $4.12 \mathrm{~mm}$.

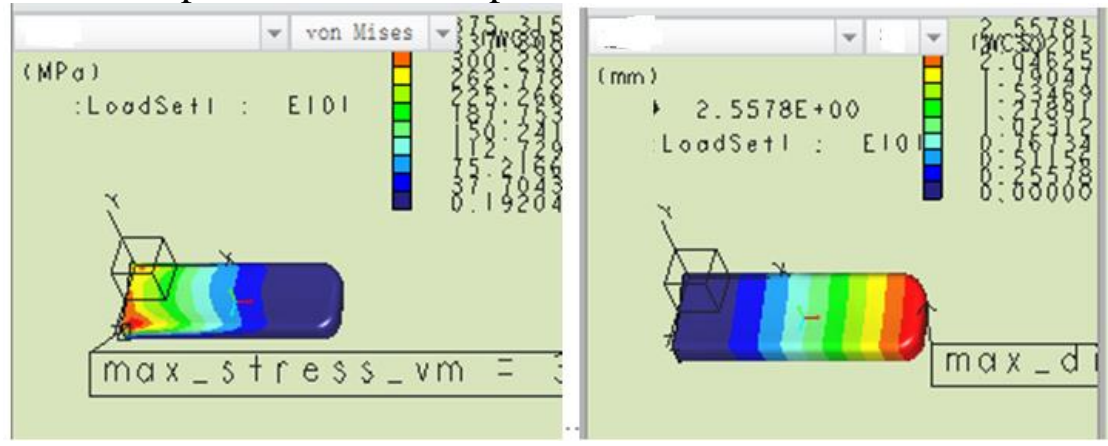

Fig.2 Von Mises distribution of blade Fig.3 Displacement distribution of blade

From the above, we can see that under different speeds, the force of fans is basically the same. The stress concentration points are all at the root of the blade, and the maximum deformation and displacement are all at the tip of the blade. The greater the speed of the blade, the greater the force of the blade, the greater the radial displacement of the blade. The maximum stress of the fan at the rated speed is $375.4 \mathrm{MPa}$, and the maximum radial displacement of the blade tip is about $2.55 \mathrm{~mm}$.

\section{Summary}

In recent years, with the popularity of computer technology and the continuous improvement of computing speed, finite element analysis has gained more and more attention in engineering design and analysis, and has become an effective way to solve complex engineering analysis and computation problems. Almost all design and manufacture can't be separated from the finite element analysis. Through the analysis and calculation, the optimization design scheme is adopted to reduce the cost of raw materials; The experiment scheme is simulated to reduce the test times and reduce the cost of the test.

\section{References}

[1] Haijun Kou, Xi Fu, Juhong Zhang,etc. Analysis of the stress and strength of the fan blade of a turbofan engine [J]. China Science Paper. Vol.11(22) 2016,p.2562-2567 
[2] Zhenzhen Liu, Zhixiong Chen, Jin Chen. Strength Analysis of an Aeroplane Engine's Fan Blade[J]. Journal of Aerospace Science and Technology. Vol.5(3) 2017, p.143-150.

[3] CHAN S K, TUBA I S. A Finite element method for contact problems of solid bodies: Part II. Application to turbine blade fastenings [J].International Journal of Mechanical Sciences, 1971,13(7): 627-639.

[4] Houlin Liu, Huan $\mathrm{Xu}$, Xianfang $\mathrm{Wu}$, etc. Strength analysis of a diffuser pump based on fluid-structure interaction[J]. Journal of Vibration and Shock.Vol.32(12), 2013 , p.27-30.

[5] Xiao Chen, Xiaobing Chen, Yan Gong. Finite element analysis of the impeller of multi airfoil centrifugal fan based on ANSYS [J]. Agricultural Sciences of JiangSu. Vol.45(9), 2017. p.180-183. 